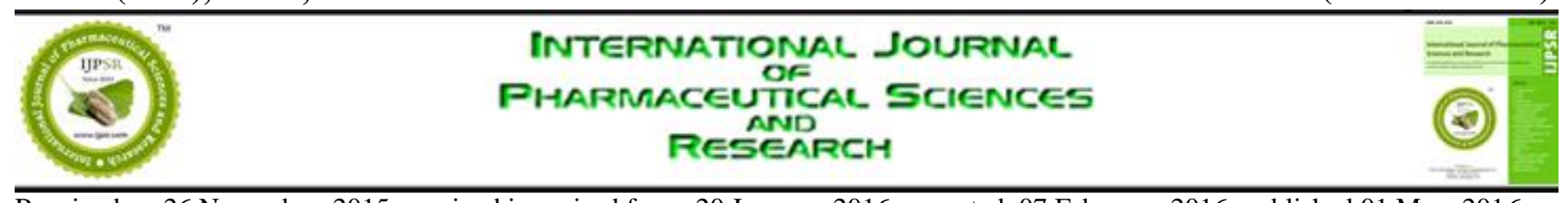

Received on 26 November, 2015; received in revised form, 20 January, 2016; accepted, 07 February, 2016; published 01 May, 2016

\title{
ROLE OF VITAMIN D IN DIABETES MELLITUS
}

Abhishek Acharya, and Susheela Somappa Halemani *

Subbaiah Institute of Medical Sciences, Shimoga, Karnataka - 577222, India

Keywords:

Vitamin D, Diabetes Mellitus, Glycemic control, Insulin

\section{Correspondence to Author:}

Dr. Susheela Somappa Halemani

w/o Dr. Abhishek Achraya

"LAXMI", 'A' Block, 1st Parallel

Road, Gandhinagar, Shimoga-

Karnataka - 577222, India .

E-mail: susheelahalemani@yahoo.com
ABSTRACT: Diabetes Mellitus (DM), a global health care problem has been a burden to the socioeconomic society. The increasing incidence highlights the need for innovative approaches for the prevention and management, despite availability of various therapies for managing disease and its complications. There are several factors that seem to play a role in development of DM including genetic, lifestyle, environmental and nutritional conditions. Amongst nutritional factors, Vitamin D is likely to have an important role either in glycemic control or in attenuating diabetic complications. Vitamin $\mathrm{D}$, an excellent marker of 'good' health has role in varied functions. Many of the epidemiologic studies have shown that vitamin D deficiency is associated with increased risk of chronic diseases such as cancer, cardiovascular disease, type 2 diabetes, autoimmune diseases and multiple sclerosis. Calcitriol (Vitamin D3) has reported to alter glycemic control and with some evidences pointing to its role in development of DM. Many studies done in past could not give conclusive association, hence benefit of supplementation of Vitamin D in DM patients need to be evaluated precisely.
INTRODUCTION: Type 2 Diabetes Mellitus (T2DM) is the commonly seen endocrine disorder characterized by hyperglycemia with absolute or relative insulin deficiency. As per the International Diabetes Federation, prevalence of DM in India has increased by $12-18 \%$ in urban, 3-6 \% in rural area over last 30 years. ${ }^{1}$ By the year 2030, this significant global health care problem is estimated to affect 552 million individuals worldwide. ${ }^{2}$ Although knowledge has been acquired on the etiology of diabetes, its precise etiopathogenesis is still under discussion.

\begin{tabular}{|l|c|}
\hline QUICK RESPONSE CODE & $\begin{array}{c}\text { DOI: } \\
\text { 10.13040/IJPSR.0975-8232.7(5).1881-88 }\end{array}$ \\
& $\begin{array}{c}\text { Article can be accessed online on: } \\
\text { www.ijpsr.com }\end{array}$ \\
\hline
\end{tabular}

Recently inflammatory factors, reactive oxygen species and autoimmune reactions have strongly emerged as the major pathogenic factors for diabetes. $^{3}$

Various therapies for type 2 diabetes and its complications have improved over the last few decades, but the increasing burden highlights the need for innovative approaches for the prevention and management of the disease.

Many risk factors play a role in etiopathogenesis and in glycemic control. To prevent development, sugar control, management of complications of DM, identification of environmental, easily modifiable risk factors (losing weight, physical activity) and use of simple natural remedies/supplements is the need of the hour in addition to existing therapy. 
Achieving excellent Glycemic control is crucial in the management of diabetes as well as preventing the onset of serious and life threatening complications. Progressive beta-cell dysfunction and insulin resistance can make ant diabetic agents less effective; treatment adherence is only around $60 \%$ which could be attributed to painful injections of insulin or hypoglycemic episodes due to oral ant diabetic agents.

There are several factors that seem to play a role in its development including genetic, lifestyle, environmental and nutritional conditions. Amongst nutritional factors, Vitamin D is likely to have an important role either in glycemic control or in attenuating diabetic complications.

Adiposity and physical inactivity are strong risk factors for type 2 diabetes. Smoking, low consumption of fish or alcohol, general and central adiposity, physical inactivity are also associated with low 25-hydroxy Vitamin D concentration, an active metabolite of Vitamin D, used as a clinical indicator. ${ }^{4}$

Vitamin D, a fat soluble vitamin discovered in 1922 by McCollum is derived from sun exposure i.e synthesized in skin, diet including fish milk, yogurt, orange juice and cereals. ${ }^{5}$ Hence Vitamin D deficiency could be attributed to either lack of sunlight exposure or insufficient dietary sources. It is a secosteroid synthesized in the skin by the action of ultraviolet irradiation from the sun related to bone metabolism and skeletal integrity as well. Its status is an excellent marker of 'good' health, including positive associations with young age, normal body weight and a healthy lifestyle.

It has been described as a wonder vitamin because of its possible benefits in diverse health outcomes like bone disease, coronary heart disease etc. Epidemiologic studies have shown that Vitamin D deficiency is associated with increased risk of chronic diseases such as cancer, cardiovascular disease, type 2 diabetes, autoimmune diseases, multiple sclerosis and type 1 diabetes mellitus too. 6

Vitamin D deficiency and diabetes mellitus are two common conditions which are widely prevalent across all ages, races, geographical regions and socioeconomic conditions. The common risk factors for both conditions include AmericanAfrican race, obesity, aging and low physical activity. ${ }^{7}$

Serum calcium levels, calcitriol (Vitamin D3) have reported to alter glycemic control and with some evidences pointing to their role in development of DM. ${ }^{8}$ Few cross sectional studies have shown that low serum concentration of $25(\mathrm{OH})$ D3 have an association with impaired glucose tolerance and diabetes. ${ }^{9-11}$

Vitamin D is likely to improve insulin action directly by stimulating expression of the insulin receptors in peripheral tissues and enhancing its responsiveness for glucose transport. ${ }^{12}$ Indirectly it could normalize extracellular calcium thus ensuring calcium influx through cell membranes to increase insulin secretion. ${ }^{13}$ It may mediate activation of beta-cell calcium dependent endopeptidases to produce the cleavage of proinsulin to insulin.

Systemic inflammatory mediators affecting Beta cells are also tackled by direct effect of Vitamin D on cytokines. Recently, Vitamin D has sparked widespread interest in the pathogenesis of diabetes and its complications. Evidences depict that its antioxidant effect has role in prevention of type 2 diabetes. ${ }^{14}$

The role of Vitamin D in DM is also suggested by a seasonal variation in glycemic control reported in patients with type 2 diabetes being worse in the winter which may be due to prevalence of ${ }_{13}$ hypovitaminosis D as a result of reduced sunlight.

The clue towards the role of Vitamin D in DM has been provided by various facts. Presence of Vitamin $D$ receptors on pancreatic $\beta$ cells ${ }^{15}$, expression of $1 \alpha$ hydroxylase activity at pancreatic $\beta$ cells causing activation of Vitamin D. ${ }^{16}$ Other evidences are presence of Vitamin D response element in human insulin gene promoter ${ }^{17}$, Vitamin D receptor in skeletal muscle ${ }^{18}$ and suppression of renin gene, increased transcription of insulin receptor genes by $1,25(\mathrm{OH}) \mathrm{D} .{ }^{19}$ Calbindin is activated by Vitamin D act as a 
modulator of depolarization stimulated insulin release via regulation of intracellular calcium.

Protective effects of Vitamin D in diabetes may be due to its anti-inflammatory properties, its effects on calcium and phosphorus metabolism. ${ }^{20}$ It plays role in the insulin sensitivity by regulating nuclear PPAR (Peroxisome proliferative activated receptor). ${ }^{21}$ It also attenuates the expression of proinflammatory cytokines involved in insulin resistance such as interleukins, IL-1, IL-6, TNF- $\alpha$, down regulates NF-Kb (Nuclear factor) activity. ${ }^{22}$

Additional evidence for a role of Vitamin D in type 2 diabetes comes from a large number of crosssectional studies, which have generally reported an inverse association between Vitamin D status and prevalent hyperglycemia

Though epidemiological studies demonstrate an inverse association between low serum $25(\mathrm{OH})$ Vitamin D and glucose intolerance but some intervention trials using Vitamin D have produced mixed results ${ }^{11,12}$.

Some of the observational longitudinal studies have shown an inverse association between the Vitamin D status $25(\mathrm{OH})$ D or self-reported Vitamin D intake and the development of type 2 diabetes. But some studies have revealed that glycemic control is independent of Vitamin D status.

Whether association between low 25(OH) D and $\mathrm{DM}$ is casual or not is yet to be proven.

\section{Studies:}

a. The Women's Health Initiative study, with sample of 33,951 women, did not observe any effect from Vitamin D in DM. ${ }^{23}$ Major limitations with this study were, low vitamin D3 dose of 400 IU/day, less than ideal compliance; and the presence of contamination, since control subjects were able to take Vitamin $1991{ }^{24}$

b. In 1984, a study done by Nilas L showed that treatment with Vitamin D or its analogues at a dose of 2,000 IU/day did not change body weight or blood glucose level in postmenopausal women, but only 25 people were on this dose. ${ }^{25}$

c. The study by Forouhi et al. reported that the baseline serum $25(\mathrm{OH})$ D levels are inversely associated with glucose and insulin levels collected 10 years later. 26 Finnish cohort study attenuated after adjustment for confounders like obesity and low physical activity showed an inverse association between baseline serum $25(\mathrm{OH}) \mathrm{D}$ and 17-year risk of type 2 diabetes. ${ }^{27}$

Together, study done by Forouhi et. al and Finnish cohort study, provided strong evidence that low Vitamin D status predicts hyperglycemia.

d. Robert Scragg's commentary on Vitamin D and Type 2 Diabetes provides new prospective evidence that low levels of Vitamin D also predict hyperinsulinemia, and suggests that Vitamin D may act to prevent type 2 diabetes by decreasing insulin resistance, although it may also inhibit insulin secretion. ${ }^{28} 2008$

e. A post hoc analysis of a trial designed for bone-related outcomes, found that $700 \mathrm{IU} /$ day of Vitamin D3 (combined with calcium) decreased homeostasis model assessment of insulin resistance in participants with impaired glucose tolerance but not in those with normal fasting glucose. ${ }^{29}$

f. With the aim to find unconfounded results, many investigators by using a Mendelian randomization approach, have examined previous observational studies to find causal associations of $25(\mathrm{OH}) \mathrm{D}$ with risk of type 2 diabetes. Unfortunately they were unable to confirm or refute causality because of small sample sizes, few included SNPs, or the absence of validation. Even issues like definite dose, need for combination treatment with calcium, compliance, and generalisability were not considered. ${ }^{30-32}$

g. The results of Mendelian randomisation analysis suggests that genetically predicted low concentrations of $25(\mathrm{OH}) \quad \mathrm{D}$ were not 
associated with risk of type 2 diabetes. This finding suggests that the association between $25(\mathrm{OH}) \mathrm{D}$ concentration and type 2 diabetes might not be causal (panel). ${ }^{33}$

h. A study done by Kostoglou-Athanassiou I. et. al showed that Vitamin D levels may have an inverse relationship with $\mathrm{HbA} 1_{C}$ and low levels of Vitamin D show a correlation to increased incidence of type 2 diabetes. Vitamin D supplementation should be considered in those with type 2 diabetes when clinically indicated. $^{34}$

i. Using data from several studies covering thousands of people of European descent, Dr. Forouhi and colleagues investigated the link between levels of Vitamin D and risk of developing diabetes by examining the genes that control blood levels of Vitamin D. The researchers did not find any evidence of low Vitamin D causing type 2 diabetes, nor they found a link between the risk of developing type 2 diabetes and the different gene variants that control blood levels of Vitamin D. ${ }^{35}$

j. According to the article published in Lancet Diabetes \& Endocrinology, October 2014 issue, the association between 25(OH)D concentration and type 2 diabetes is unlikely to be causal. Efforts to increase 25(OH)D concentrations might not reduce the risk of type 2 diabetes as would be expected on the basis of observational evidence. ${ }^{36}$

k. In a recent study, by de Boer et al examined the effect of calcium plus Vitamin D supplementation on the incidence of drugtreated diabetes in postmenopausal women and concluded that, calcium plus Vitamin D3 supplementation did not reduce the risk of developing diabetes over seven years of follow-up in this randomized, placebo controlled trial. ${ }^{37}$

1. Diabetes has been associated with several neurological disorders including reduced locomotor activity which in turn is associated with low concentration of Vitamin D. A study by K.T. Peeyush reported the altered expression of cholinergic and dopaminergic receptors in the central nervous system of STZ-induced diabetic rats. They further suggested that the altered expression of these receptors was brought back to control by the treatment with Vitamin D. ${ }^{38,39}$

m. A small, clinical study of 51 patients with type 2 diabetes (37 female) evaluated neuropathic complaints such as pain, burning, tingling, numbness, and throbbing sensations. Patients who were vitamin D deficient at base line were given cholecalciferol (D3) and re-evaluated at 3-month follow-up. Serum concentrations increased $67.4 \%$ from 18 to $30 \mathrm{ng} / \mathrm{ml}$, and were associated with significantly lower pain scores. 40

These findings provide a confirmatory evidence for neuroprotective role of Vitamin D and represent a novel possibility for the better management of diabetic mediated complications.

n. Nurses' Health Study that included 83,779 women $>20$ years of age found an increased risk of type 2 diabetes in those with low Vitamin D status. A combined daily intake of $>800 \mathrm{IU}$ of Vitamin D and $1,000 \mathrm{mg}$ of calcium reduced the risk of type 2 diabetes by $33 \%{ }^{41}$

It is possible that optimal levels of serum Vitamin D may be different for people at risk for developing diabetes, those with diabetes, and those without diabetes.

o. A Japanese study evaluating 581 diabetic patients and 51 nondiabetic ones showed a prevalence of hypovitaminosis $\mathrm{D}(<20 \mathrm{ng} / \mathrm{mL})$ of $75 \%$ with no differences between the type 2 diabetic patients and the control group. ${ }^{41}$

p. Von Hurst et al. supplemented the diets of nondiabetic overweight South Asian women with $4000 \mathrm{IU} / \mathrm{d}$ Vitamin $\mathrm{D}_{3}$ for 6 months and found a significant improvement in insulin sensitivity compared with a placebo group. ${ }^{42}$ 
q. A study of 83,779 women with no history of diabetes over 2-4 years showed that a daily combined intake of $1200 \mathrm{mg}$ of $\mathrm{Ca}$ and $800 \mathrm{IU}$ of Vitamin D was associated with 33\% lower risk of type 2 diabetes as compared with a daily intake of less than $600 \mathrm{mg}$ of $\mathrm{Ca}$ and less than 400 IU of Vitamin .

DISCUSSION: Based on the results of the Diabetes Control and Complications Trial, hyperglycemia is an important contributor to the development of diabetic nephropathy in type 1 diabetes, and maintenance of euglycemia can prevent or delay progression of renal disease. Link between the observations that maintenance of euglycemia and blockade of the RAAS prevent or delay diabetic kidney disease remains entirely hypothetical. ${ }^{43}$

Since Vitamin D can enhance insulin sensitivity, suppress renin gene, it can be hypothesized to have an effect on diabetic control and better management of complications.

Dr. Neil Binkley gave a presentation at the 1 . Southern Regional Conference of the ADA in May of 2010. According to Binkley's report, diabetes is one of many diseases/conditions associated with low Vitamin D status. In fact, he hypothesized that low Vitamin D may play a role in the development of diabetes. Potential mechanisms underlying this association are supported by the following findings. 8,44

Vitamin D improves beta cell function

-Direct effect on insulin secretion $-1,25(\mathrm{OH}) 2 \mathrm{D}$ stimulates insulin release -its supplementation restores impaired insulin4. secretion in Vitamin D deficient mice5. -Indirect effect via calcium on insulin secretion hypocalcemia is associated with impaired insulin6. secretion

Vitamin D is known to improve insulin action -It stimulates expression of the insulin receptor -It enhances insulin responsiveness for glucose8. transport.

Reducing hyperglycemic induced increase in renin levels in pancreatic $\beta$ cells and blockade of renin-

2 . angiotensin activity has been proposed as a novel target for diabetes treatment. ${ }^{45}$

\section{Role of Vitamin D in Inflammation:}

It is currently recognized that t2DM is associated with systemic inflammation which has been linked to insulin resistance but elevated cytokines may also play a role in beta cell dysfunction by triggering beta cell apoptosis. Vitamin D may improve insulin sensitivity and promote beta-cell survival by directly modulating the generation and effects of cytokines. ${ }^{46}$

Nearly three decades have passed since the studies linking Vitamin D with insulin metabolism have begun, but none of them provide convincing conclusion. A well-designed clinical trial to evaluate the effect of Vitamin D supplementation on glycemia status and diabetes risk is urgently required to settle this question.

Deficiencies of previous studies need to overcome, which include

Confounding factor: Low physical activity, adiposity might confound the association substantially. Increased adiposity, lowers 25(OH)D concentration and increases the risk of type 2 diabetes as well. Measurements of confounders (eg, physical activity) are susceptible to errors and are not adequately controlled in epidemiological analysis

3. Subclinical conditions such as liver disease can lower production of $25(\mathrm{OH}) \mathrm{D}$ as well as increase the risk of type 2 diabetes suggesting reverse causality $(\mathrm{K})$, hence to be ruled out. ${ }^{47}$

Lack of well-designed clinical trials- small sample size, lack of generalisability, poor compliance.

7. Foods rich in Vitamin D may have an effect on glycemic status or replace other foods that might increase risk of type 2 diabetes. Uniform food habits should be part of study design.

9. Definite dose proves to be helpful in DM is not yet defined. In particular, the Vitamin D dose given in such trials needs to be high enough - above 2,000 IU per day to raise blood $25(\mathrm{OH}) \mathrm{D}$ levels above 80 
$\mathrm{nmol} / \mathrm{l}$ because diabetes risk is lowest at this level. 48,49

CONCLUSION: Although the role of Vitamin D in helping to regulate blood glucose remains poorly understood, many of the previous cross sectional studies have shown that Vitamin D status appears to play a role in the development and treatment of diabetes. It is possible that optimal levels of serum Vitamin D may be different for people at risk for developing diabetes, those with diabetes, and those without diabetes.

According to Danescu et al, "both animal and human studies support the notion that adequate Vitamin D supplementation may decrease the incidence of type 1 and possibly also of type 2 diabetes mellitus and may improve the metabolic control in the diabetes state. However, the exact mechanisms are not clear and need further investigation." 50

Overall, observational longitudinal studies have shown an inverse association between the Vitamin D status $(25(\mathrm{OH}) \mathrm{D}$ or self-reported Vitamin D intake) and the development of type 2 diabetes. In RCTs, Vitamin D supplementation did not show any beneficial effects on glycemic measures among persons with normal glucose tolerance but there were beneficial effects among patients with glucose intolerance or insulin resistance at baseline.

The challenge for health care providers and nutrition researchers is to determine whether Vitamin D deficiency actually causes or increases the incidence of certain diseases or whether, instead low levels of Vitamin D are simply coincidental given that the majority of the general population, regardless of disease, is likely to have insufficient levels of Vitamin D.

Since Vitamin D can enhance insulin sensitivity by various actions, suppress renin gene which is responsible for hyperglycemia induced complications, it can be hypothesized to have an effect on prevention, diabetic control and better management of complications.

Larger studies are needed to assess more precisely the potential causal association between $25(\mathrm{OH}) \mathrm{D}$ concentrations and risk of type 2 diabetes. If causality does exist, interventions such as sunlight exposure or increased Vitamin D intake (diet or supplementation) could provide a simple, inexpensive, and safe prevention strategy for type 2 diabetes.

Various short-term studies (follow up $\leq 3$ months) suggested that Vitamin D supplementation has a positive impact on glycemic control and metabolic parameters such as insulin resistance and beta cell dysfunction.

However, the evidence was weak due to the low methodological quality of the studies. There was no significant effect on $\mathrm{HbAlc}$, beta cell function and insulin resistance in the long-term studies (follow up > 3 months). There existed heterogeneity in the methodology of the studies, inclusion criteria, mode of supplementation of Vitamin D and the duration of follow up.

Large-scale trials with proper study design, optimal Vitamin D supplementation and longer follow up need to be conducted.

ACKNOWLEDGEMENT: I thank Dr. Mahesh G.K, Dr. Siddalingappa. C.M, Mrs. Vagdevi, Dr. Anand, my collegues from Department of Pharmacology, Subbaiah Institute of Medical Sciences, Shimoga, for their timely help and support.

\section{REFERENCES:}

1. Balasubramanian $\mathrm{S}$, Carnagarin $\mathrm{R}$, Jagdeeshwaran $\mathrm{A}$, Parthasarathy J P, Thatiparthi S. Serum 25(OH)D and Type 2 Diabetes Mellitus. J Clin Diagn Res 2012; 6:774-6.

2. IDF: One adult in ten will have diabetes by 2030 . IDF Press release; 2011 [http://www.idf.org/mediaevents/press-releases/2011/diabetes-atlas-5th-edition], accessed on 29th August 2014

3. Tuorkey MJ, Abdul-Aziz KK. Strategies for diabetes and pathways of vitamin D: Diabetes and Metabolic Syndrome: Clinical Research and Reviews 2010;4:101110.

4. Kuhn T, Kaaks R, Teucher B, et al. Dietary, lifestyle, and genetic determinants of vitamin $\mathrm{D}$ status: a cross-sectional analysis from the European Prospective Investigation into Cancer and Nutrition (EPIC)-Germany study. Eur J Nutr 2014; 53:731- 41

5. Holick MF. Sunlight, vitamin D and health: A D-lightful story. Oslo: The Norwegian Academy of Science and Letters. Espen Bjertness. 2008. 
6. Luiz Henrique, Maciel Griz. Vitamin D and diabetes mellitus: an update - 2013, Arq Bras Endocrinol Metab 2014;58/1

7. Alvarez JA, Ashraf A: Role of vitamin D in insulin secretion and insulin sensitivity for glucose Homeostasis. Int J Endocrinal 2010:351-85.

8. Pittas AG, Lau J, Hu FB, Hughes BD. The Role of Vitamin D and Calcium in Type 2 Diabetes: A Systematic Review and Meta- Analysis. J Clin Endocrinol Metab 2007;92:2017-29.

9. Scragg R, Holdaway I, Singh V, Metcalf P, Baker J, Dryson E. Serum 25-hydroxyvitamin D3 levels decreased in impaired glucose tolerance and diabetes mellitus. Diabetes Res Clin Pr 1995;27:181-8.

10. Isaia $\mathrm{G}$, Giorgino $\mathrm{R}$, Adami $\mathrm{S}$. High prevalence of hypovitaminosis $\mathrm{D}$ in female type 2 diabetic population (Letter). Diabetes Care 2001;24:1496.

11. Scragg R, Sowers M, Bell C. Serum 25- hydroxyvitamin $\mathrm{D}$, diabetes, and ethnicity in the Third National Health and Nutrition Examination Survey. Diabetes Care 2004;27:2813-18

12. Mathieu C, Gysemans C. Vitamin D and diabetes. Av Diabetol 2006;22:187-93.

13. Mathieu C, Gysemans C, Giulietti A, Bouillon R. Vitamin D and diabetes. Diabetologia 2005;48:1247-57.

14. Krishna G Seshadri, Role of Vitamin D in Diabetes, J Endocrinol Metab 2011;1:47-56.

15. Zittermann A: Vitamin D and disease prevention with special reference to cardiovascular disease. Prog Biophys Mol Biol 2006;92:39- 48.

16. Bland R, Markovic D, Hills CE, Hughes SV, Chan SL, Squires PE, et al. Expression of 25-hydroxyvitamin D31alpha- hydroxylase in pancreatic islets. J Steroid Biochem Mol Biol 2004;89:121-125.

17. Maestro B, Davila N, Carranza MC, Calle C. Identification of a Vitamin D response element in the human insulin receptor gene promoter. J Steroid BiochemMol Biol 2003;84:223-30.

18. Simpson RU, Thomas GA, Arnold AJ: Identification of 1, 25-dihydroxyvitamin D3 receptors and activities in muscle. J Biological Chemistry 1985;260:8882- 91.

19. Maestro B, Molero S, Bajo S. Transcriptional activation of the human insulin receptorgene by 1,25 -dihydroxyvitamin D(3). Cell bio-chemfunct 2002;20:227-32.

20. Ojuka E. Role of calcium AMP kinase in the regulation of mitochondrial biogenesis and GLUT4 levels in muscle. Proc Nutr soc 2004;63:275-78.

21. Dunlop TW, Vaisanen S, Frank C, Molnar F, Sinkkonen L, Carlberg $\mathrm{C}$, et al. The human peroxisome proliferatoractivated receptor $\delta$ gene is a primary target of $1 \alpha, 25$ dihydroxyvitamin D3 and its nuclear receptor. J Molecular Biology 2005; 349:248-60.

22. Cohen-Lahav M, Douvdevani A, Chaimovitz C, Shany S. The anti inflammatory activity of 1,25 dihydroxy vitamin D3 in macrophages. J Steroid Biochem Mol Biol 2007; 103:558-62.

23. Boer IH, Tinker LF, Connelly S, Curb JD, Howard BV, Kestenbaum B et al. Calcium plus vitamin D supplementation and the risk of incident diabetes in the Women's Health Initiative. Diabetes Care 2008;31:701-7.

24. Heaney RP, Davies KM, Chen TC, Holick MF, BargerLux MJ. Human serum 25-hydroxycholecalciferol response to extended oral dosing with cholecalciferol. Am J Clin Nutr 2003; 77:204- 10.

25. Nilas L, Christiansen C. Treatment with vitamin D or its analogues does not change body weight or blood glucose level in postmenopausal women. Int J Obes 1984; 8:40711.

26. Forouhi NG, Luan J, Cooper A, Boucher BJ, Wareham N. Baseline 25-hydroxy vitamin $\mathrm{D}$ is predictive of future glycemic status and insulin resistance: the Medical Research Council Ely prospective study. Diabetes2008; 57:2619- 25.

27. Mattila C, Knekt P, Mannisto S, Rissanen H, Laaksonen MA, Montonen J, Reunanen A: Serum 25-hydroxyvitamin D concentration and subsequent risk of type 2 diabetes. Diabetes Care 2007;30:2569-70.

28. Robert Scragg: Vitamin D and Type 2 Diabetes. Are We Ready for a Prevention Trial?: a commentary. DIABETES 2008;57:2565-6.

29. Pittas AG, Harris SS, Stark PC, Dawson-Hughes B. The effects of calcium and vitamin D supplementation on blood glucose and markers of inflammation in nondiabetic adults. Diabetes Care 2007;30:980- 6.

30. Jorde R, Schirmer H, Wilsgaard T. Polymorphisms related to the serum 25-hydroxyvitamin $\mathrm{D}$ level and risk of myocardial infarction, diabetes, cancer and mortality. The Troms $\varnothing$ Study. PLoS One. 2012;7: e37295. [PubMed: 22649517]

31. Buijsse B, Boeing H, Hirche F. Plasma 25-hydroxyvitamin $\mathrm{D}$ and its genetic determinants in relation to incident type 2 diabetes: a prospective case-cohort study. Euro J Epidemiol 2013;28:743-52.

32. Afzal S, Brøndum-Jacobsen P, Bojesen SE, Nordestgaard BG. Vitamin D concentration, obesity, and risk of diabetes: a mendelian randomisation study. Lancet Diabetes Endocrinol 2014;2:298-06. [PubMed: 24703048]

33. Zheng Ye, Stephen J Sharp. Association between circulating 25-hydroxyvitamin $\mathrm{D}$ and incident type 2 diabetes: a mendelian randomisation study. Lancet Diabetes Endocrinol. 2015; 3:35- 42.

34. Kostoglou-Athanassiou. Vitamin D and Glycemic Control in Diabetes Mellitus Type 2. Therapeutic Advances in Endocrinology and Metabolism 2013;4:122-8.

35. Low Vitamin D and Type 2 Diabetes, Diet, BG Control, Type 2 Diabetes, Issue 704

36. 36. The Lancet Diabetes \& Endocrinology, early online publication, 1 October 2014, Association between circulating 25-hydroxyvitamin $\mathrm{D}$ and incident type 2 diabetes: a Mendelian randomisation study. doi:10.1016/S2213-8587(14)70184-6

37. de Boer IH, Tinker LF, Connelly S, Curb JD, Howard BV Kestenbaum B, Larson JC, et al. Calcium plus vitamin D supplementation and the risk of incident diabetes in the Women's Health Initiative. Diabetes Care 2008;31:701-7.

38. Calle C, Maestro B, Garcia-Arencibia M. Genomic actions of 1,25-dihydroxyvitamin D3 on insulin receptor gene expression, insulin receptor number and insulin activity in the kidney, liver and adipose tissue of streptozotocininduced diabetic rats. BMC Mol Biol 2008;9:65.

39. Peeyush KT, Savitha B, Sherin A, Anju TR, Jes P, Paulose CS. Cholinergic, dopaminergic and insulin receptors gene expression in the cerebellum of streptozotocin- induced diabetic rats: functional regulation with Vitamin D3 supplementation. Pharmacol Biochem Behav 2010;95:21622

40. Lee P, Chen R. Vitamin D as an analgesic for patients with type 2 diabetes and neuropathic pain. Arch Intern Med 2008; 68:771-2.

41. Pittas AG, Dawson-Hughes B, Li T, Van Dam RM, Willett WC, Manson JE, Hu FB: Vitamin D and calcium intake in 
relation to type 2 diabetes in women. Diabetes Care 2006;29:650-6.

42. Von Hurst PR, Stonehouse W, Coad J. Vitamin D supplementation reduces insulin resistance in South Asian women living in New Zealand who are insulin resistant and vitamin D deficient- a randomized placebo-controlled trial. Br J Nutr 2010;103:549 5 [PubMed: 19781131]

43. Diabetes Control and Complications Trial Research Group. The effect of intensive treatment of diabetes on the development and progression of long-term complications in insulin dependent diabetes mellitus. N Engl J Med 1993; 329:977-86.

44. European Journal of Clinical Nutrition (2011) 65, 10051015; doi:10.1038/ejen.2011.118; published online 6 July 2011.

45. Cheng Q, Li YC, Boucher BJ, Leung PS. A novel role for vitamin D: modulation of expression and function of the local renin-angiotensin system in mouse pancreatic islets. Diabetologia 2011; 54:2077-81.
46. Anastassios G. Pittas, MD MSc. The Role of Vitamin D and Calcium in type 2 diabetes. A systematic Review and Meta-Analysis* J Clin Endocrinol Metab 2007; 92:201729

47. Kwok RM, Torres DM, Harrison SA. Vitamin D and nonalcoholic fatty liver disease (NAFLD): is it more than just an association? Hepatology 2013;58:116674.[PubMed: 23504808]

48. Vieth R, Bischoff-Ferrari H, Boucher BJ, Dawson-Hughes $\mathrm{B}$, Garland CF, Heaney RP, Holick MF et al. The urgent need to recommend an intake of vitamin $\mathrm{D}$ that is effective. Am J Clin Nutr 2007;85:649-50.

49. Scragg R, Holdaway I, Singh V, Metcalf P, Baker J, Dryson E. Serum 25-hydroxyvitamin D3 levels decreased in impaired glucose tolerance and diabetes mellitus. Diabetes Res Clin Pract 1995;27:181-8.

50. Danescu LG, Levy S, Levy J. Vitamin D and diabetes mellitus. Endocr 2009;35:11-17.

How to cite this article:

Acharya A and Halemani SS: Role of Vitamin D in Diabetes Mellitus. Int J Pharm Sci Res 2016; 7(5): 1881-88.doi: 10.13040/IJPSR.09758232.7(5).1881-88.

All @ 2013 are reserved by International Journal of Pharmaceutical Sciences and Research. This Journal licensed under a Creative Commons Attribution-NonCommercial-ShareAlike 3.0 Unported License.

This article can be downloaded to ANDROID OS based mobile. Scan QR Code using Code/Bar Scanner from your mobile. (Scanners are available on Google Playstore) 